\title{
New Potential Drug Candidates Against SARS-CoV-2 Using Generative Model
}

\author{
Madhusudan Verma* \\ Deep Learning Engineer at Pucho Life Science Inc \\ madhusudan@pucholifesciences.com
}

\begin{abstract}
Since known approved drugs like liponavir and ritonavir failed to cure SARS-CoV-2 infected patients, it is high time to generate new chemical entities against this virus. $3 C L$ main protease acts as key enzyme for the growth of a virus which acts as biocatalyst and helps to break protein and ultimately in the growth of coronavirus. Based on a recently solved structure (PDB ID: 6LU7), we developed a novel advanced deep Q-learning network with the fragment-based drug design $(A D Q N-F B D D)$ along with variational autoencoder with KL annealing and circular annealing for generating potential lead compounds targeting SARS-CoV-2 3CL pro. Structure-based optimization policy (SBOP) is used in reinforcement learning. The reason for using variational autoencoders is that it does not deviate much from actual inhibitors, but since VAE suffers from KL diminishing we have used KL annealing and circular annealing to address this issue. Researchers can use this compound as potential drugs against SARS-CoV-2.
\end{abstract}

\section{Introduction}

SARS-CoV2 coronavirus has caused pandemic worldwide which causes disease Covid-19. The number of cases and deaths is increasing day by day. This virus is similar to SARS-Covid for which there is no approved treatment. The virus becomes deadly when it causes severe acute respiratory syndrome. So, there is an urgent need to create inhibitors for this. This approach works by adding fragments instead of atoms which is not only computationally efficient but also chemically more reasonable. The rate of a reaction is defined by $r=k e^{-E_{a} \mid R T}$ where $\mathrm{k}$ is rate constant, $E_{a}$ is the activation energy, $\mathrm{R}$ molar gas constant and $\mathrm{T}$ is the absolute temperature.

When the kinetic energy of the reactant molecules is greater than the activation energy, the reaction occurs. The reaction between inhibitor and protease is given by:

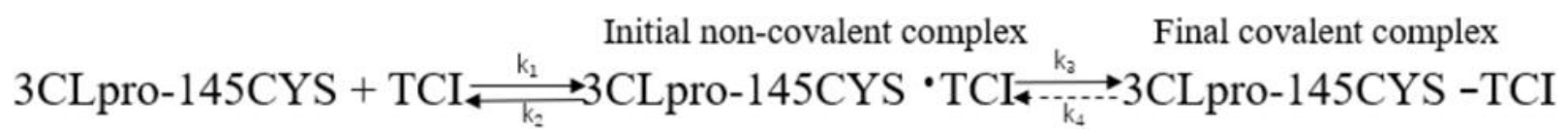


$\mathrm{k} 1, \mathrm{k} 2, \mathrm{k} 3$ and $\mathrm{k} 4$ are the rate constants. For inhibition to occur k1 must be greater than $\mathrm{k} 2$ for non-covalent bonding. After this step covalent bonding takes place such that $\mathrm{k} 3>>\mathrm{k} 4$ and the reverse reaction does not happen. We generated molecules using Variational Autoencoder using transfer learning on inhibitors, then applied reinforcement learning by adding fragments and calculating reward, so that molecules with the desired property are obtained which includes drug likeness, specific fragment containment and pharmacophores scores.

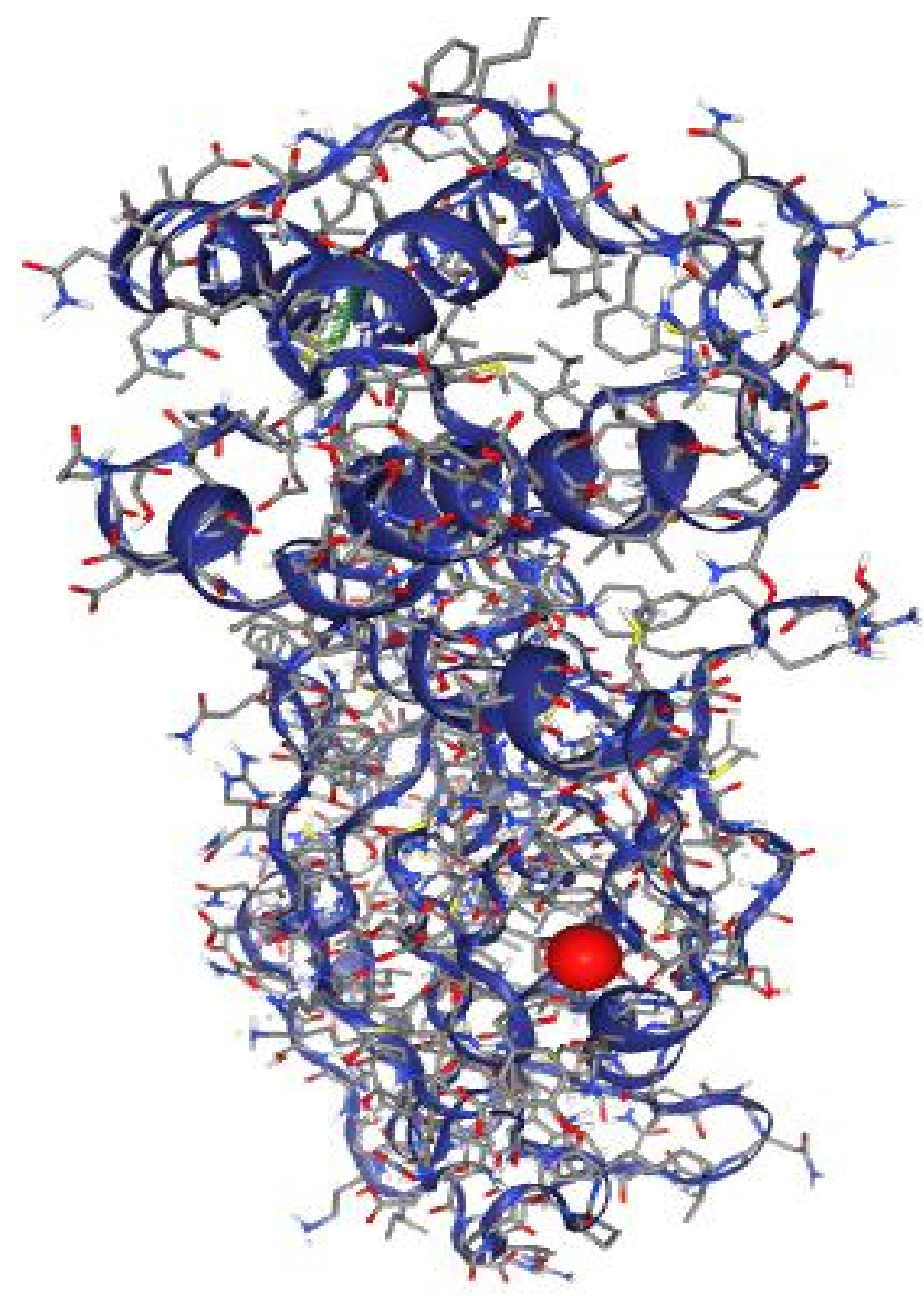

Figure1. SARS-CoV-2 main protease with unliganded active site.

Keywords: COVID-19, SARS-COV2, 3CL Protease, Structure-based optimization policy, Deep learning, Artificial intelligence, Reinforcement learning. 


\section{Related Work}

Bowen Tang, Fengming He, Dongpeng Liu, Meijuan Fang, Zhen Wu have used Reinforcement learning on SARS-CoV-2 3CLPro main protease inhibitors and defined reward function which includes other properties like fragment containment and pharmacophores score and applied efficient and novel advanced deep Q-learning network with the fragment-based drug design.

Navneet Bung, Sowmya Ramaswamy Krishnan, Gopalakrishnan Bulusu and Arijit Roy from Tata Consultancy Services Limited have trained RNN on 1.6 million data points from $\mathrm{ChEBL}$ dataset to learn the grammar of smile representation of molecules ,then used transfer learning on 2515 inhibitors from the same dataset and applied reinforcement learning to force generator generate molecules with desired Synthetic accessibility score, quantitative estimate of drug-likeness, partition coefficient and molecular weight.

IBM has generated 3000 novel molecules using generative models against three targets. In addition, the Hong Kong-based pharmaceutical research company, InSilico Medicine has rolled out a list of 97 potential candidate molecules designed to inhibit the $3 \mathrm{CL}$ protease of SARS-CoV-2.

\section{Results}

Table1:Docking Results using Covid19 Docking Server(which uses AutodockVina program) with $3 \mathrm{CL}$ protease as target

\begin{tabular}{|l|l|}
\hline Molecule & Score \\
\hline $\mathrm{m} 1$ & -8.40 \\
\hline $\mathrm{m} 2$ & -7.80 \\
\hline $\mathrm{m} 3$ & -8.20 \\
\hline M3 & -8.10 \\
\hline
\end{tabular}

Description:The scoring function that combines certain advantages of knowledge-based potentials and empirical scoring functions.

Table2:Docking Results using Covid19 Docking Server(which uses AutodockVina program) with RdRp(RTP site) as Target 


\begin{tabular}{|l|l|}
\hline Molecule & Score \\
\hline $\mathrm{m} 1$ & -9.0 \\
\hline $\mathrm{m} 2$ & -8.40 \\
\hline $\mathrm{m} 3$ & -10.0 \\
\hline M3 & -8.80 \\
\hline
\end{tabular}

Table3:Docking results of generated molecules with 3CL main protease.

\begin{tabular}{|l|l|l|l|l|}
\hline Molecules & Affinity & $\begin{array}{l}\text { Total } \\
\text { Energy }\end{array}$ & $\begin{array}{l}\text { VdW } \\
\text { Energy }\end{array}$ & $\begin{array}{l}\text { Elec. } \\
\text { Energy }\end{array}$ \\
\hline $\mathrm{m} 1$ & -8.815 & 55.511 & -26.251 & -4.554 \\
\hline $\mathrm{m} 2$ & -8.786 & 32.002 & -25.460 & -10.323 \\
\hline $\mathrm{m} 3$ & -8.401 & 44.086 & -20.373 & -8.075 \\
\hline $\mathrm{M} 3$ & -8.537 & 28.338 & -21.283 & -12.288 \\
\hline
\end{tabular}

Table4:Druglikeness properties according to Lipinki's rule

\begin{tabular}{|l|l|l|l|l|}
\hline Id & Mw(g/mol) & Logp(Concensus) & HBA & HBD \\
\hline $\mathrm{m} 1$ & 421.49 & 3.85 & 3 & 0 \\
\hline $\mathrm{m} 2$ & 447.53 & 2.16 & 6 & 3 \\
\hline $\mathrm{m} 3$ & 454.42 & 0.17 & 8 & 3 \\
\hline $\mathrm{M} 3$ & 438.49 & 3.90 & 5 & 2 \\
\hline
\end{tabular}


Table:5 Water Solubility

\begin{tabular}{|l|l|l|l|}
\hline Id & $\begin{array}{l}\text { Log S (ES } \\
\text { OL) }\end{array}$ & Solubility & Class \\
\hline $\mathrm{m} 1$ & -5.21 & $2.58 \mathrm{e}-03 \mathrm{mg} / \mathrm{ml}$ & $\begin{array}{l}\text { Moderately } \\
\text { soluble }\end{array}$ \\
\hline $\mathrm{m} 2$ & -4.61 & $1.17 \mathrm{e}-02 \mathrm{mg} / \mathrm{ml}$ & $\begin{array}{l}\text { Moderately } \\
\text { soluble }\end{array}$ \\
\hline $\mathrm{m} 3$ & -3.26 & $2.48 \mathrm{e}-01 \mathrm{mg} / \mathrm{ml}$ & Soluble \\
\hline $\mathrm{M} 3$ & -4.92 & $5.27 \mathrm{e}-03 \mathrm{mg} / \mathrm{ml}$ & $\begin{array}{l}\text { Moderately } \\
\text { soluble }\end{array}$ \\
\hline
\end{tabular}

Table4:Docking results of HIV protease inhibitors with 3CL main protease.

\begin{tabular}{|l|l|l|l|l|}
\hline Molecules & Affinity & $\begin{array}{l}\text { Total } \\
\text { Energy }\end{array}$ & $\begin{array}{l}\text { VdW } \\
\text { Energy }\end{array}$ & $\begin{array}{l}\text { Elec. } \\
\text { Energy }\end{array}$ \\
\hline Ritonavir & $-\mathbf{8 . 8 8 7}$ & 32.378 & -25.152 & -13.400 \\
\hline Darunavir & $-\mathbf{8 . 1 3 0}$ & 11.724 & -20.395 & -13.779 \\
\hline Lopinavir & -7.707 & 73.229 & -18.948 & -15.515 \\
\hline
\end{tabular}

Table 5: Generated Molecules with high Tanimoto Similarity with the already existing 3CL Main Protease Inhibitors of SARS-CoV2.

\begin{tabular}{|l|l|l|l|}
\hline ID & MOLECULES & 3CL MAIN PROTEASE & Tanimoto \\
\hline
\end{tabular}




(INHIBITORS OF




(1)

Table 6: Drug Likeness Properties of the Generated Molecule and satisfying Drug likeness properties

\begin{tabular}{|l|l|l|l|l|}
\hline ID. & MW & $\log \mathbf{P}$ & HBA & HBD \\
\hline m1 & 421.19 & 4.67 & 5 & 0 \\
\hline
\end{tabular}




\begin{tabular}{|l|l|l|l|l|}
\hline m2 & 438.2 & 3.63 & 4 & 2 \\
\hline M_1 & 417.37 & 3.733 & 8 & 4 \\
\hline M_2 & 412.61 & 5.73 & 3 & 0 \\
\hline M_3 & 476.53 & 4.00 & 6 & 2 \\
\hline M_4 & 272.61 & 3.43 & 3 & 1 \\
\hline M_5 & 278.27 & 2.18 & 4 & 1 \\
\hline M 6 & 298.29 & 2.97 & 5 & 2 \\
\hline M_7 & 426.51 & 3.35 & 3 & 2 \\
\hline M_8 & 466.92 & 4.29 & 5 & 2 \\
\hline M_9 & 281.27 & 1.89 & 5 & 0 \\
\hline PM_1 & 495.63 & 3.956 & 8 & 1 \\
\hline
\end{tabular}

MW - Molecular Weight; Log P - Partition coefficient; HBA - Hydrogen Bond Acceptor; HBD - Hydrogen Bond Donor

\section{Discussion}

If the activation energy $E_{a}$, which is the difference in energy between reactants and activated complex is less, which is also called the Gibbs energy of activation represented by the equation,

\section{$\Delta \mathrm{G}^{\ddagger}=\Delta \mathrm{H}^{\ddagger}-\mathrm{T} \Delta \mathrm{S}^{\ddagger}$}

then the rate of the reaction is more, assuming temperature is constant. So, if the rate of reaction in forward direction is more in both the steps of inhibitor reaction then inhibition will take place. So, we can reward the agent if the molecule formed after adding fragments has less activation energy and penalize if it has higher activation energy. Further, Variational Autoencoder can be improved. The conventional VAE uses KL divergence as a regularization term, instead of this, Wasserstein Distance can be used which is better than KL divergence. We can also use a generalized version of 
log likelihood and KL divergence similar to Coupled-VAE. Conditional GANs where QED score, fragment containment scores and pharmacophores are used as conditional input can also be used.

\section{Methods}

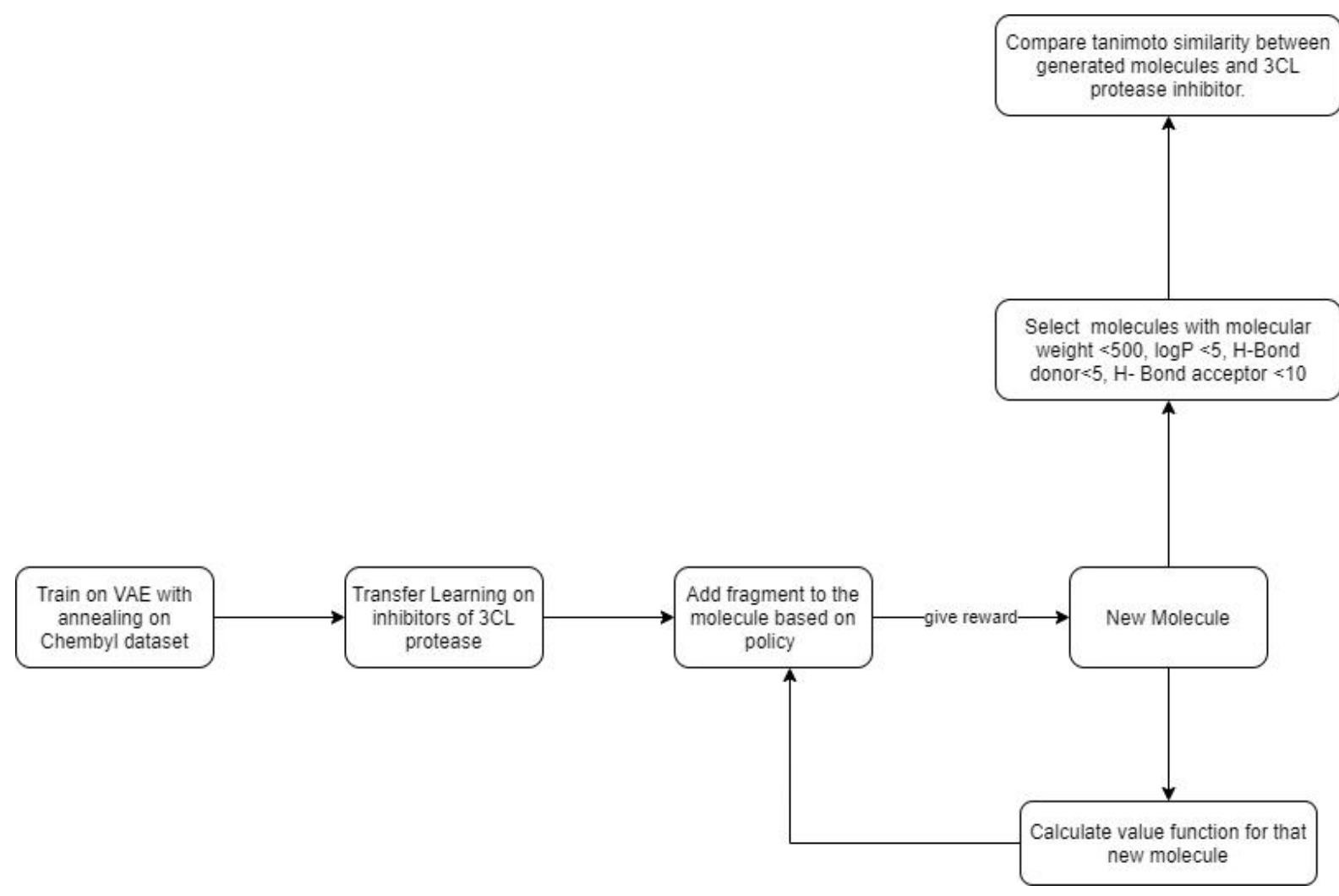

Figure2: Flow diagram for the method

\subsection{Variational Autoencoder With KL Divergence And Circular Annealing}

Given a datapoint $\mathrm{x}$, the goal of VAE is to find at least one latent vector which is able to describe it; one vector that contains the instructions to generate $x$. If we formulate it using the law of total probability, we get $P(x)=\int P(x \mid z) P(z) d z$. The VAE training objective is to maximize $P(x) \cdot P(x \mid z)$ is modeled using a multivariate Gaussian $\mathrm{N}\left(\mathrm{f}(\mathrm{z}), \sigma^{2} * \mathrm{I}\right)$ where $\sigma^{2}$ is the hyperparameter that will be multiplied by I which represents the identity matrix. $f(z)$ is modeled using a neural network.

The formula for $P(x)$ is intractable, so it is approximated using Monte Carlo method which is described below: 
1. Sample $\left\{z_{i}\right\}_{i=1}^{n}$ from the prior $P(z)$.

2. Approximate using $P(x) \approx \frac{1}{n} \sum_{i=1}^{n} p\left(x \mid z_{i}\right)$.

Since most sampled z's won't contribute anything to $\mathrm{P}(\mathrm{x})$, therefore, a new distribution $Q(z \mid x)$ is introduced. $Q(z \mid x)$ is trained to give high probability values to $z$ 's that are likely to have generated $x$. Now the Monte Carlo estimation can be calculated using much fewer samples from $Q(z \mid x)$.

Since

$\log (t) \leq t-1$

$-\mathrm{D}_{\mathrm{KL}}(\mathrm{q}(\mathrm{x}) \| \mathrm{p}(\mathrm{x}))=\int \mathrm{q}(\mathrm{x}) \log \left(\frac{p(x)}{q(x)}\right) d x \leq \int \mathrm{q}(\mathrm{x})\left(\frac{p(x)}{q(x)}-1\right) d x$

$\leq \int \mathrm{p}(\mathrm{x}) d x-\int \mathrm{q}(\mathrm{x}) d x=1-1=0$

$\Rightarrow \mathrm{D}_{\mathrm{KL}}(\mathrm{q}(\mathrm{x}) \| \mathrm{p}(\mathrm{x})) \geq 0$

This means

$D_{K L}\left((p(z \mid x) \| q(z \mid x))=\int p(z \mid x) \log \left(\frac{p(z \mid x)}{q(z \mid x)}\right) d x>=0\right.$

Applying Bayes' theorem we get 


$$
\begin{aligned}
& -\int q(z \mid x) \log \left(\frac{p(x \mid z) p(z)}{q(z \mid x) p(x)}\right) d z \geq 0 \\
& \Rightarrow-\int q(z \mid x)\left[\log \left(\frac{p(x \mid z) p(z)}{q(z \mid x)}\right)-\log (p(x))\right] d z \geq 0 \\
& \Rightarrow-\int q(z \mid x) \log \left(\frac{p(x \mid z) p(z)}{q(z \mid x)}\right) d z+\int q(z \mid x) \log (p(x)) d z \geq 0 \\
& \Rightarrow-\int q(z \mid x) \log \left(\frac{p(x \mid z) p(z)}{q(z \mid x)}\right) d z+\log (p(x)) \int q(z \mid x) d z \geq 0 \\
& \Rightarrow-\int q(z \mid x) \log \left(\frac{p(x \mid z) p(z)}{q(z \mid x)}\right) d z+\log (p(x)) \geq 0 \\
& \Rightarrow \log (p(x)) \geq \int q(z \mid x) \log \left(\frac{p(x \mid z) p(z)}{q(z \mid x)}\right) d z \\
& \Rightarrow \log (p(x)) \geq \int q(z \mid x) \log \left(\frac{p(x \mid z) p(z)}{q(z \mid x)}\right) d z \\
& \Rightarrow \log (p(x)) \geq \int q(z \mid x) \log \left(\frac{p(z)}{q(z \mid x)}\right) d z+\int q(z \mid x) \log (p(x \mid z) d z \\
& \Rightarrow \log (p(x)) \geq-D_{K L}(q(z \mid x) \| p(z))+E_{z \sim q(z \mid x)}[\log (p(x \mid z)]
\end{aligned}
$$

We took $\log (p(x))$ out of integral because if $\mathrm{x}$ is given then $\log p(x)$ will be constant, so maximizing log likelihood which is intractable is the same as minimizing its lower bound. Since it is not possible to differentiate with respect to random variable reparameterization trick is used if we sample $\varepsilon$ from standard normal distribution and substitute $z=\mu+\varepsilon * \sigma$.

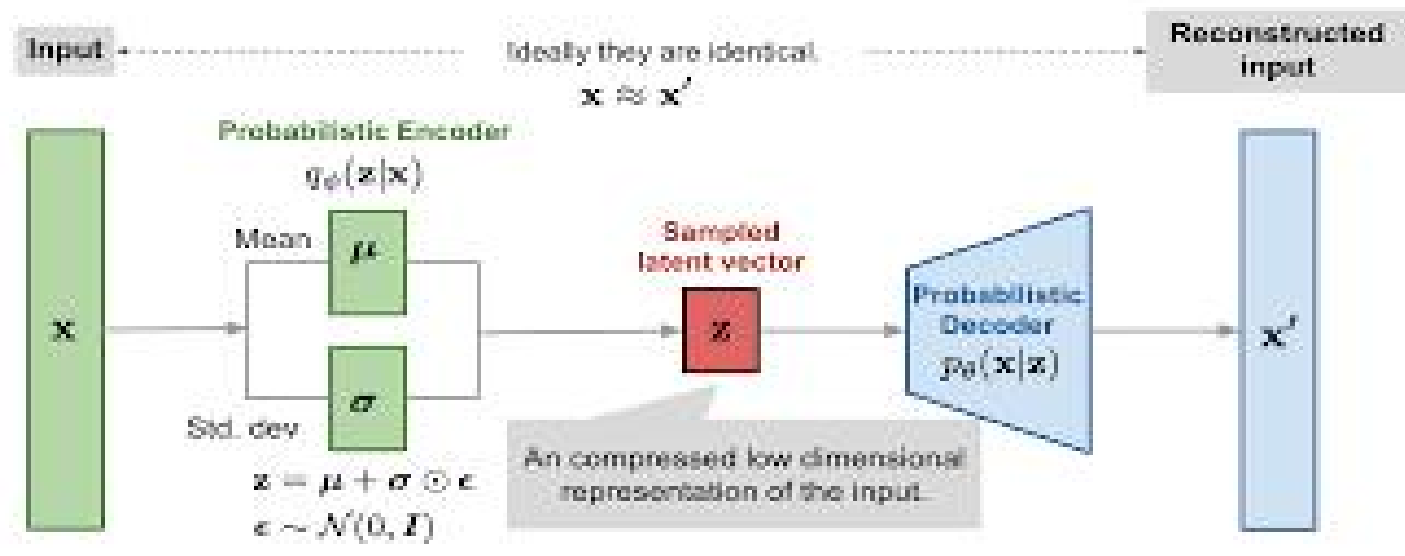

Figure 3. Vanilla VAE 
The loss function consists of negative likelihood along with a regularizer term and a hyperparameter $\beta$ which is increased slowly in case KL annealing and periodically in case of circular annealing. This solves KL diminishing problem which makes VAE to act as simple RNN.

$$
\operatorname{Loss}(\theta, \varphi)=-E_{z \sim q_{\theta}(x)}\left[\log \log \left(p_{\varphi}(x \mid z)\right]+\beta K L\left(q_{\theta}(z \mid x) \| p(z)\right)\right.
$$

\subsection{Markov Reward Process}

This is an extension of Markov decision process for Reinforcement learning. This consists of states, actions, transition probability, rewards and discounting factors. In our case state will be the molecule structure at time step t will be state and adding and removing fragments will be action. Here instead of decreasing discounting factor for higher time step, it is increased by using $\lambda^{T-t}$ where $T$ is the time step at the end of the episode.

\subsection{Agent Design}

Agent fits the $Q^{\pi}\left(s_{t}, a_{t}\right)$ function which is the cumulative reward starting from state at time step $t$ choosing action based on policy $\pi$ such that it has maximum cumulative reward using advanced deep Q learning. The state-action and the value of the state are defined as

$$
\begin{aligned}
& Q^{\pi}\left(s_{t}, a_{t} ; \theta, \alpha, \beta\right)=E_{a_{t} \sim \pi\left(s_{t}\right)}\left[\sum_{n=t}^{T} \gamma^{T-n} \cdot R\left(s_{n}, a_{n}\right)\right] \\
& =E_{a_{t} \sim \pi\left(s_{t}\right)}\left[R\left(s_{t}, a_{t}\right)+\gamma E_{a_{t} \sim \pi\left(s_{t+1}\right)}\left(Q^{\pi}\left(s_{t+1}, a_{t+1}\right)\right]\right.
\end{aligned}
$$

$$
V^{\pi}\left(s_{t}\right)=E_{a_{t} \sim \pi\left(s_{t}\right)}\left[Q^{\pi}\left(s_{t}, a_{t}\right)\right]
$$

In order to make RL more stable and to handle the problem of overestimation of Q-values a duel DQN network as target network is used. We keep its parameters $\theta^{\prime}, \alpha^{\prime}, \beta^{\prime}$ fixed and copy from dueling DQN $Q^{\pi}$ every $m$ steps.

$$
T D=Q^{\pi}\left(s_{t}, a_{t} ; \theta, \alpha, \beta\right)-\left[R\left(s_{t}{ }^{\prime}, a_{t}\right)+\gamma \cdot Q_{t a r}{ }^{\pi}\left(s_{t+1}, \arg \max _{a_{t+1}} Q^{\pi}\left(s_{t+1}, a_{t}+1 ; \theta, \alpha, \beta\right) ; \theta^{\prime}, \alpha^{\prime}, \beta^{\prime}\right)\right]
$$

Q function depends on parameters which come from dueling Q networks. Temporal difference is used in which reward is given at time step instead of end of episode. The parameters of Dueling Q network are updated as

$$
\operatorname{loss}(\theta, \alpha, \beta)=E[f(T D)]
$$

$\mathrm{RL}$ agent is trained to minimize this loss function where $\mathrm{f}$ is the hubber function. 


\subsection{Reward Design}

$$
R(s)=w_{\text {pro }} f(s)_{\text {pro }}+w_{c s f} f(s)_{c s f}+w_{\text {pha }} f(s)_{\text {pha }}
$$

Pro represents QED which is drug likeness property, csf represents whether a particular fragment is present and pha stands for pharmacophores score which depends on ligand-protein interaction mode from the crystal structure.

\section{$\underline{\text { References }}$}

1) Bowen Tang, Fengming He, Dongpeng Liu, Meijuan Fang, Zhen Wu.AI-aided design of novel targeted covalent inhibitors against SARS-CoV-2. BioRxiv(2020)

2) Hao Fu1 $\uparrow$, Chunyuan Li2 $\uparrow *$, Xiaodong Liu2 Jianfeng Gao2, Asli Celikyilmaz2, Lawrence Carin 1.Cyclical Annealing Schedule: A Simple Approach to Mitigating KL Vanishing

3) Gorbalenya AE. Severe acute respiratory syndrome-related coronavirus-The species and its viruses, a statement of the Coronavirus Study Group. BioRxiv, (2020). Google Scholar

4) Coronavirus COVID-19 Global Cases by Johns Hopkins CSSE, available from: https://forum.truckersmp.com/index.php?/topic/93003-coronavirus-covid-19-glob al-cases-by-johns-hopkins-csse/\&tab=comments. Google Scholar

5) Zhang L, et al. Alpha-ketoamides as broad-spectrum inhibitors of coronavirus and enterovirus replication Structure-based design, synthesis, and activity assessment. Journal of Medicinal Chemistry, (2020). Google Scholar

6) Liu X, Wang X-J. Potential inhibitors for $2019-\mathrm{nCoV}$ coronavirus $\mathrm{M}$ protease from clinically approved medicines. bioRxiv, (2020). Google Scholar

7) Yang $\mathrm{H}$, et al. Design of wide-spectrum inhibitors targeting coronavirus main proteases. PLoS biology 3, (2005). Google Scholar

8) Singh J, Petter RC, Baillie TA, Whitty A. The resurgence of covalent drugs. Nature reviews Drug discovery 10, 307-317 (2011). CrossRefPubMedGoogle Scholar

9) Tuley A, Fast W. The taxonomy of covalent inhibitors. Biochemistry 57, 3326-3337 (2018). Google Scholar

10) $\mathrm{Wu} \mathrm{C}-\mathrm{Y}$, et al. Small molecules targeting severe acute respiratory syndrome human coronavirus. Proceedings of the National Academy of Sciences 101, 10012-10017 (2004).Abstract/FREE Full TextGoogle Scholar

11) Ghosh AK, et al. Design and synthesis of peptidomimetic severe acute respiratory syndrome chymotrypsin-like protease inhibitors. Journal of medicinal chemistry 48, 6767-6771 (2005). CrossRefPubMedWeb of ScienceGoogle Scholar

12) Shie J-J, et al. Discovery of potent anilide inhibitors against the severe acute respiratory syndrome $3 \mathrm{CL}$ protease. Journal of medicinal chemistry 48, 4469-4473 (2005).CrossRefPubMedGoogle Scholar 
13) Shie J-J, et al. Inhibition of the severe acute respiratory syndrome $3 \mathrm{CL}$ protease by peptidomimetic $\alpha, \beta$-unsaturated esters. Bioorganic $\&$ medicinal chemistry 13 , 5240-5252 (2005). Google Scholar

14) Al-Gharabli SI, et al. An efficient method for the synthesis of peptide aldehyde libraries employed in the discovery of reversible SARS coronavirus main protease (SARS-CoV Mpro) inhibitors. ChemBioChem 7, 1048-1055 (2006). PubMedGoogle Scholar

15) Lu I-L, et al. Structure-based drug design and structural biology study of novel nonpeptide inhibitors of severe acute respiratory syndrome coronavirus main protease. Journal of medicinal chemistry 49, 5154-5161 (2006). CrossRefPubMedGoogle Scholar

16) Tsai K-C, et al. Discovery of a novel family of SARS-CoV protease inhibitors by virtual screening and 3D-QSAR studies. Journal of medicinal chemistry 49, 3485-3495 (2006). CrossRefPubMedGoogle Scholar

17) $\mathrm{Wu} \mathrm{C}$-Y, et al. Stable benzotriazole esters as mechanism-based inactivators of the severe acute respiratory syndrome 3CL protease. Chemistry \& biology 13, 261-268 (2006). CrossRefPubMedGoogle Scholar

18) Akaji K, Konno H, Onozuka M, Makino A, Saito H, Nosaka K. Evaluation of peptide-aldehyde inhibitors using R188I mutant of SARS 3CL protease as a proteolysis-resistant mutant. Bioorganic \& medicinal chemistry 16, 9400-9408 (2008). Google Scholar

19) Ghosh AK, et al. Design, synthesis and antiviral efficacy of a series of potent chloropyridyl ester-derived SARS-CoV 3CLpro inhibitors. Bioorganic \& medicinal chemistry letters 18, 5684-5688 (2008).Google Scholar. 